\title{
Inclination of Fast Changing Currents Effect the Readings of Static Energy Meters
}

\author{
Bas ten Have ${ }^{1}$, Tom Hartman ${ }^{1}$, Niek Moonen ${ }^{1}$, Frank Leferink ${ }^{1,2}$ \\ ${ }^{1}$ University of Twente, Enschede, The Netherlands, bas.tenhave@utwente.nl \\ ${ }^{2}$ THALES Nederland B.V., Hengelo, The Netherlands
}

\begin{abstract}
Static energy meters can be forced to give misreadings due to conducted electromagnetic interference (EMI). In previous research cases lower and higher readings of static energy meters were observed. In this paper an overview of previously reported lab experiments is given and further analyzed. The various situations are showing errors in the energy readings with respect to a reference meter. Based on these findings measurements are done using a dimmer in combination with a series of compact fluorescent lightning (CFL) and light emitting diode (LED) lamps. This setup was powered using a non-distorted mains power supply created by a four-quadrant amplifier combined with a line impedance stabilization network (LISN) to create a stable line impedance. The setup creates a pulsed current waveform with a short rise time. By using various line inductances the slope of the pulse is lowered and a correlation between the inclination of the slope and the deviations of the static meters is shown.
\end{abstract}

\section{INTRODUCTION}

Electronic devices used by consumers in a low-voltage distribution network have a non-linear behavior, these electronic devices include: switched mode power supplies in computers or televisions, rectifiers in chargers, and lamps [1]. These non-linear devices can create conducted EMI [2]. The use of these devices is increasing, while the use of conventional linear (resistive) equipment is decreasing. Conducted EMI can cause static energy meters to deviate, as was shown in previous research. Static energy meters are used to measure the energy consumption in household situations [3], [4]. These meters are called smart meters if a communication link is added. The objective of smart meters is to make use of the (price) dynamics in future smart grids. In [2], a difference of $+40 \%$ in energy readings was observed between two farmers that installed the same photo voltaic (PV) system. This was caused by the power drives of fans, resulting in high interference levels. In [5], [6], active infeed converters connected to PV systems resulted in lower readings of static meters. Controlled lab experiments have shown faulty energy readings of static meters when these are loaded with equipment creating pulsed currents. The experiments show that when static meters are loaded with a dimmer and a series of CFL and LED lamps, deviations between $-46 \%$ and $+276 \%$ [7], and $-32 \%$ and $+582 \%$ [8], [9], can arise. High energy readings were also observed by a consumer, who used a commercially available water pump. Lab experiments showed energy deviations between $-61 \%$ and $+2675 \%$, [10]. It was observed that the grid impedance and the phase shift of the current influences the deviations measured by static energy meters. Additional measurements showed that currents with short rise time created by a water pump could result in deviations between $-91 \%$ and $+175 \%$ [11] .

In this paper these reported lab experiments that show deviations of static meters are analyzed more thoroughly, and additional measurements were done that show a correlation between the inclination of the current pulse and the deviations of the static meters. These experiments are performed with a series of CFL and LED lamps combined with a dimmer. The inclination of the slope is lowered by adding various inductances in series. The experiments are performed using an ideal power supply with a standardized mains impedance. The corresponding current slopes are expressed and the inclination is correlated with the misreadings of the meters. The purpose of the paper is to show that the amount of inclination a fast changing current pulse has is correlated to the readings of static meters.

The rest of this paper is organized as follows: In Section II the theoretical concepts used to compare current waveforms are explained. Section III analyzes the previous reported lab experiments on the deviations of static meters, it summarizes the effect of the grid impedance, phase shift and current slope on the deviations. Section IV describes the static meter measurement setup that is used and Section V shows the results of the measurements performed with the static meter setup, meter deviations, attributed waveforms, and corresponding slopes. These results are discussed in Section VI, and a correlation between the steepness of the current slopes and the static meter deviations is shown. Finally, in Section VII it is concluded that a steeper slope of the current pulse resulted in higher deviations of the static meters.

\section{THEORY}

In this section the theoretical concepts used to describe different current waveforms are explained. This gives a more thorough comparison between current waveforms obtained from different loads in comparable measurements.

\section{A. Slope inclination}

The inclination of the current slope is determined for the waveforms measured and analyzed in this paper, which is the ratio between the rising edge amplitude and rise time. The rise time is the time needed to rise from $10 \%$ to $90 \%$ of the peak value, which is used as a measure for fast rise times in electronics [12]. Two different variations of the waveform are considered, see Fig. 1, the signal rises to the peak value in one 
(signal type 1) or in two steps (signal type 2). In the cases considered in this paper, both signal types have comparable maximum peak values. Since the objective is to compare the inclination of the slope, for signal type 2 the slope is calculated for the first and steepest part of the inclination. But this means that the corresponding rising edge and rise time cannot be compared for two different variations of the signal, because for both situations the $10 \%$ to $90 \%$ values as indicated in Fig. 1 are used. The waveforms could have multiple peaks in one period, the absolute value of the signal is taken, and the maximum slopes per signal are considered in the analysis.

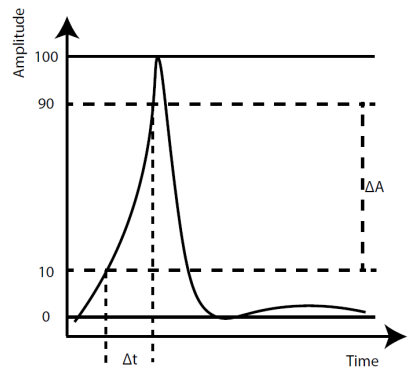

(a) Signal type 1

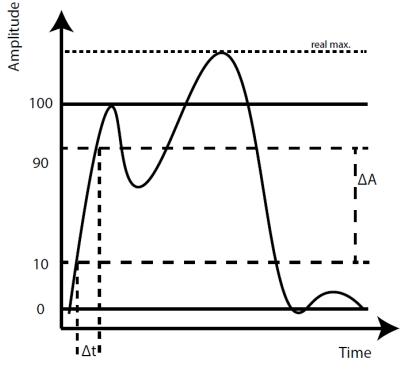

(b) Signal type 2
Fig. 1: The two different signal types considered.

\section{B. Charge}

Another method to compare current waveforms is to look at the area bounded by the current, or the charge. This is a useful method to compare signals with comparable inclinations, but a difference in phase shift, such that it is clear that the only difference between the compared signals is the phase shift. The charge, $\mathrm{Q}$, is determined using eq. 1, where $\mathrm{N}$ is the total number of samples, $i$ is the current, and $\Delta t$ is the time between two samples. Compared with the current slope this gives a good view of the similarity of the signals.

$$
Q[C]=\sum_{n=1}^{N} i(n) \Delta t
$$

By applying the above stated analyses to previous reported experiments, it is expected that a better comparison between the obtained signals related to the static meter deviations can be made, this is shown in the next section.

\section{ANALYSIS}

This section analyzes previous reported lab experiments on the deviations of static meters, which is the actual difference with respect to the reference divided by the reference value. It shows the effect of current slope, phase shift and grid impedance on the meter deviations.

\section{A. Current slope}

The lab experiments described in [8] and [9] have shown static meter deviations when CFL and LED lamps are used with a dimmer, while the setup is powered using a buildings mains supply. When the dimmer is turned to $135^{\circ}$ a slope of $1.1 \mathrm{~A} / \mu \mathrm{s}$ is generated and static meter deviations between $-32 \%$ and $+582 \%$ are registered. When the phase shift introduced by the dimmer is reduced to $90^{\circ}$, the slope reduces to $0.67 \mathrm{~A} / \mu$ s and the deviations reduce to values between $-28 \%$ and $+64 \%$. The current slope was reduced further by inserting a $1.2 \mathrm{mH}$ line choke in series with the load, this results in a much lower current slope of $0.086 \mathrm{~A} / \mu \mathrm{s}$, reducing the deviation to below $\pm 3 \%$. The corresponding current waveforms can be seen in Fig. 2.

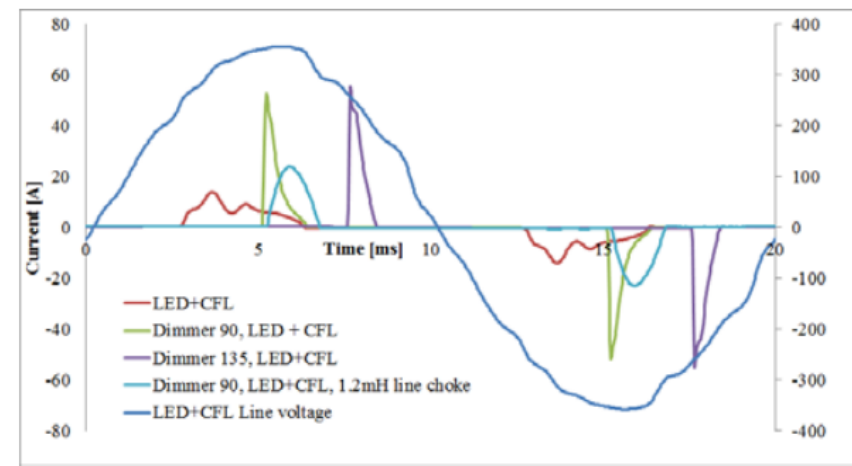

Fig. 2: Current waveforms generated by CFL and LED lamps combined with a dimmer [8].

In [11] experiments were performed using power (speed) regulators in conjunction with a commercially available water pump as a load for a series of static meters. These experiments were performed using an ideal power supply with a standardized mains impedance. Using this setup different waveforms are generated with small current spikes, that differ in slope. The corresponding waveform with regulator 1 (as it is indicated in the paper) can be seen in Fig. 3. The corresponding rise time, rising edge and slope of the current can be seen in Table I. The regulator had three settings, ranging from low to high speed. Increasing the speed from low to medium to high, results in reducing the current waveform's slopes. And the meter deviations reduced from between $-91 \%$ and $+3 \%$, to between $-72 \%$ and $10 \%$ and finally to $\pm 10 \%$ respectively. These measurements were repeated with slightly different power regulators, which showed similar results, as can be seen in [11].

TABLE I: Rise time, rising edge and slope of current spikes, for different speed levels of the regulator (Reg) [11].

\begin{tabular}{|l|r|r|r|}
\hline Reg speed level & Rise time $[\mu \mathrm{s}]$ & Rising edge $[\mathrm{A}]$ & Slope $[\mathrm{A} / \mu \mathrm{s}]$ \\
\hline Low & 1.5 & 16.0 & 10.7 \\
\hline Medium & 1.9 & 15.2 & 8.2 \\
\hline High & 2.1 & 4.2 & 2.0 \\
\hline
\end{tabular}

\section{B. Phase shift}

Other experiments with a commercially available water pump, using an ideal power supply with a standardized mains impedance, are conducted in [10]. Using a remote control unit, the water pump's capacity can be varied by phase shifting 


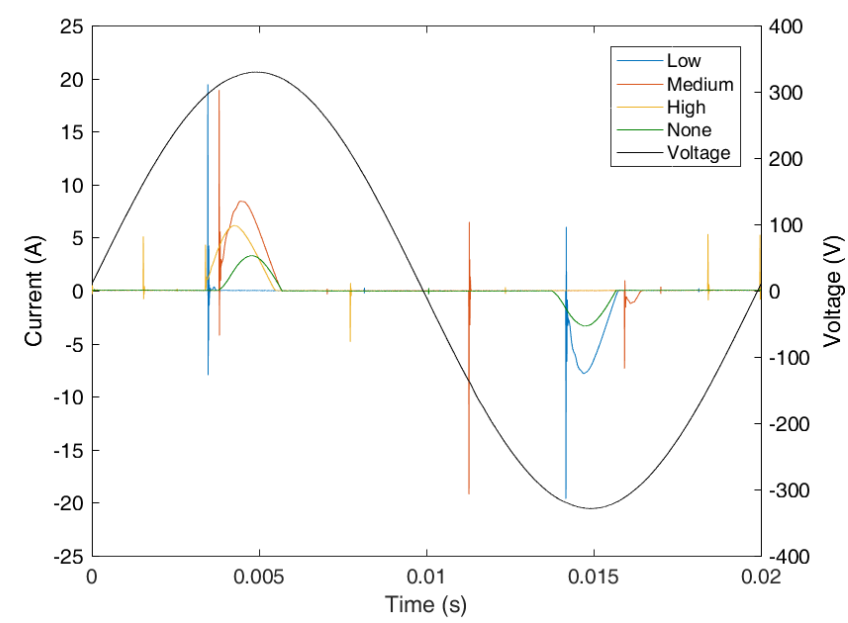

Fig. 3: Current waveforms generated by regulator 1 in combination with the water pump for the three different regulator levels, the voltage is the wave generated by the power supply without a load [11].

the current. From the recorded waveforms shown in Fig. 4, it can be seen that at full power (level 10), the phase shifter is bypassed. Table II shows the slope of the pulse and the area bounded by the current over one period, for these different capacity levels. The inclination is relatively constant with varying capacity level. Next to this, also the amount of charge per $50 \mathrm{~Hz}$ cycle is relatively constant. Fig. 5 shows the related static meter deviations. When comparing level 4 till 8, the area does not change that much, but the static meter deviations decrease. This means that it is more likely to have higher deviations if the current signal experiences more phase shift.

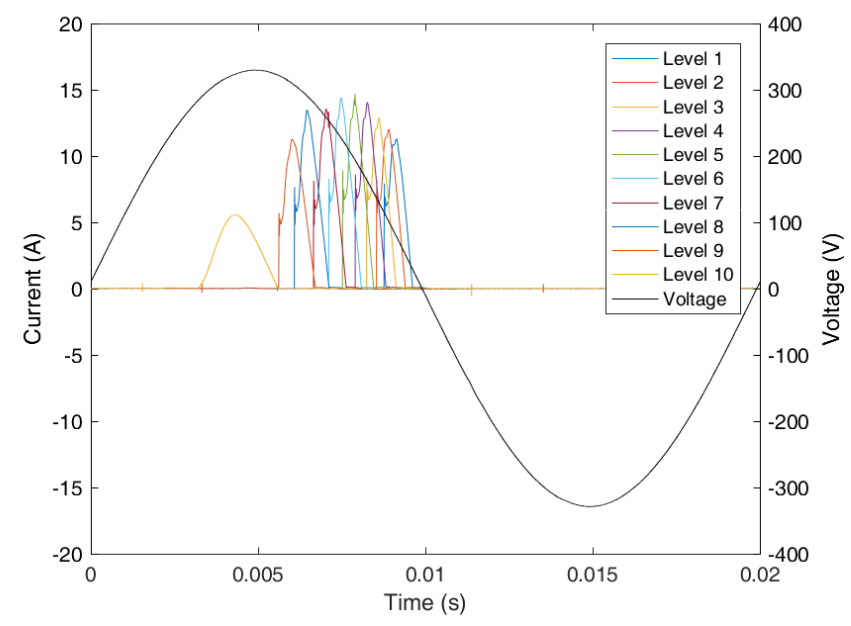

Fig. 4: Current waveforms generated by remote control in combination with the water pump, the voltage is the wave generated by the power supply without a load [10].
TABLE II: Slope of current pulse rising and area under the current wave over one period, for remote control combined with water pump for different capacity levels.

\begin{tabular}{|l|r|r|}
\hline Capacity level & Slope $[\mathrm{A} / \mu \mathrm{s}]$ & Charge $[\mathrm{mC}]$ \\
\hline 1 & 1.1 & 6.3 \\
\hline 2 & 1.1 & 6.8 \\
\hline 3 & 1.2 & 7.2 \\
\hline 4 & 1.2 & 8.0 \\
\hline 5 & 1.1 & 8.2 \\
\hline 6 & 1.1 & 8.6 \\
\hline 7 & 1.1 & 8.2 \\
\hline 8 & 1.0 & 8.5 \\
\hline 9 & 0.8 & 7.8 \\
\hline 10 & 0.01 & 7.8 \\
\hline
\end{tabular}

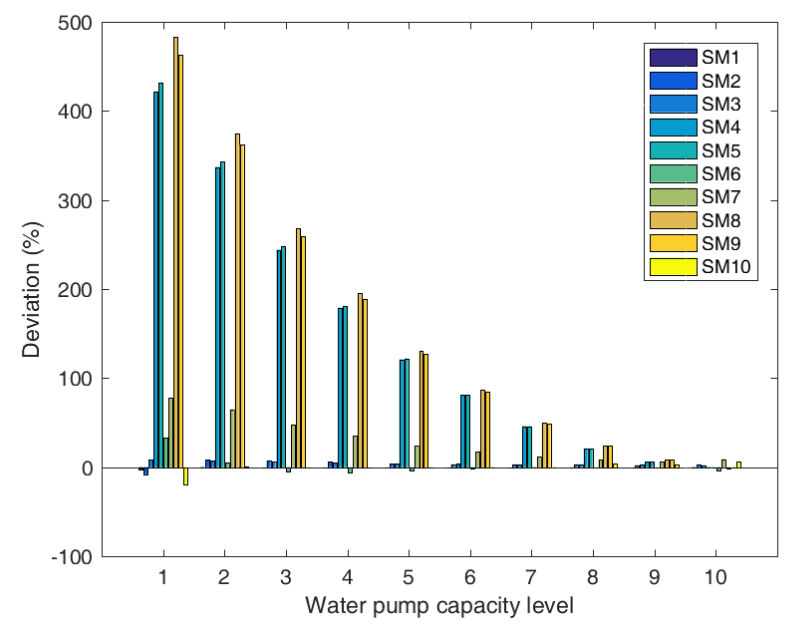

Fig. 5: Deviations [\%] between the static meters (SM) and reference, for the ten different levels of the water pump's capacity [10].

\section{Grid impedance}

Additional measurements were performed in [10] to test the effect of the grid impedance on the deviations of the static meters. Measurements are performed with different grid impedances: an ideal power supply using standard impedance, lower impedance created by the addition of a capacitance between line and neutral, higher impedance created by the addition of a series inductance, and a building's mains supply. The load is similar to the previously discussed phase shift measurements. Fig. 6 shows the deviations for different grid impedances, when the remote control is set to level 1. For a lower impedance, higher deviations are found, while a higher impedance results in lower deviations. The low impedance grid would allow the system to draw high frequency currents by the load and could explain why the grid impedance is influencing the meters readings. When a building's mains supply is used even higher deviations were found. The associated current waveforms are plotted for the remote control set to level 1 in Fig. 7. The corresponding characteristics of the pulses are shown in Table III. The waveforms show a higher amplitude when the grid impedance is decreased. Another difference is 
the total duration of the pulse, which is longer at a higher impedance, making it look more like a half wave sinusoidal. However, there is not a lot of difference in the slope of the pulse. This shows that also the rise times, the amplitude and the total duration of the pulse are of importance and extreme values will cause the meters to deviate more. These observations are similar for the other capacity levels of the remote control, [10].

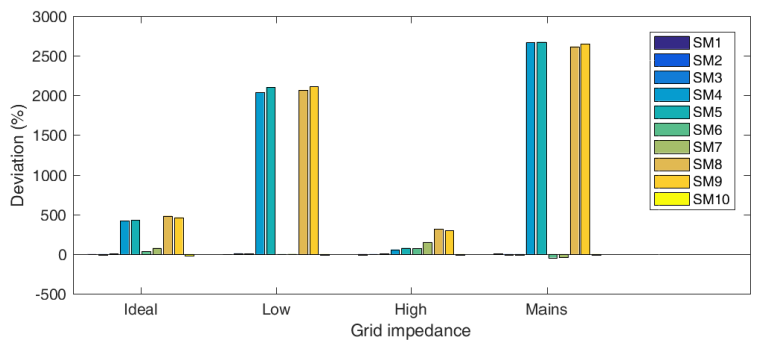

Fig. 6: Deviations [\%] between the static meters (SM) and reference, for different grid impedances [10].

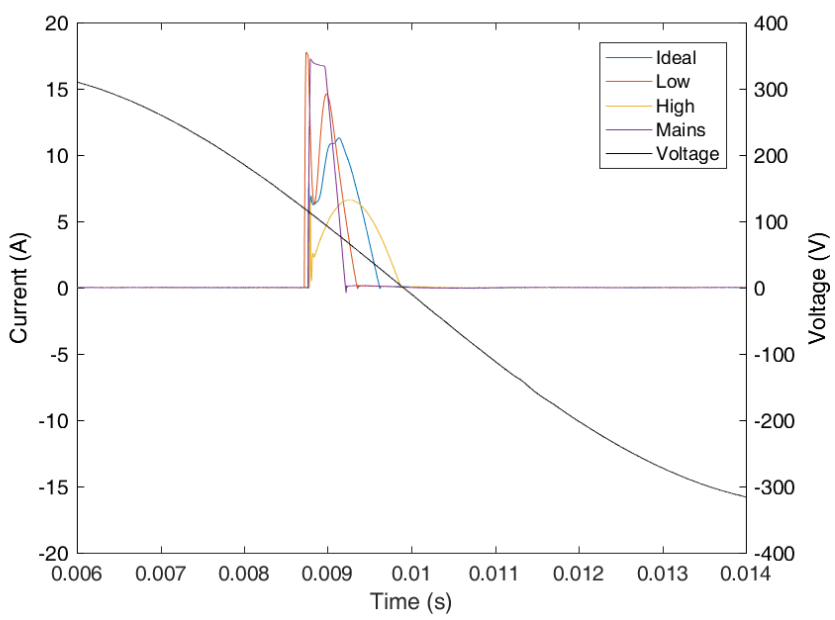

Fig. 7: Current waveforms generated, for different grid impedances, the voltage is the wave generated by the power supply without a load [10].

TABLE III: Rise time, rising edge and slope of current spikes, for different grid impedances [10].

\begin{tabular}{|l|r|r|r|}
\hline Grid impedance & Rise time $[\mu \mathrm{s}]$ & Rising edge $[\mathrm{A}]$ & Slope $[\mathrm{A} / \mu \mathrm{s}]$ \\
\hline Ideal & 5.8 & 6.3 & 1.1 \\
\hline Low & 13.8 & 14.1 & 1.0 \\
\hline High & 5.4 & 6.3 & 1.2 \\
\hline Mains & 18.8 & 13.7 & 0.7 \\
\hline
\end{tabular}

\section{Effect inclination on misreadings}

Although it was shown that the inclination of fast rising currents has an effect on static meter readings, no direct correlation between them is shown, since also other parameters were not constant. An explanation for these static meter deviations could be the low sampling rate of the static meters, [9]. In case of lower readings this could mean that part of the signal is missed by the static meter, for higher readings measured, the signal could be overestimated. Another explanation might be that the high and steep current peak, creates a huge overload for the current transducer and forces the measuring current transducer to go into saturation. Therefore, additional measurements are performed in which the inclination will be varied and other parameters are kept constant.

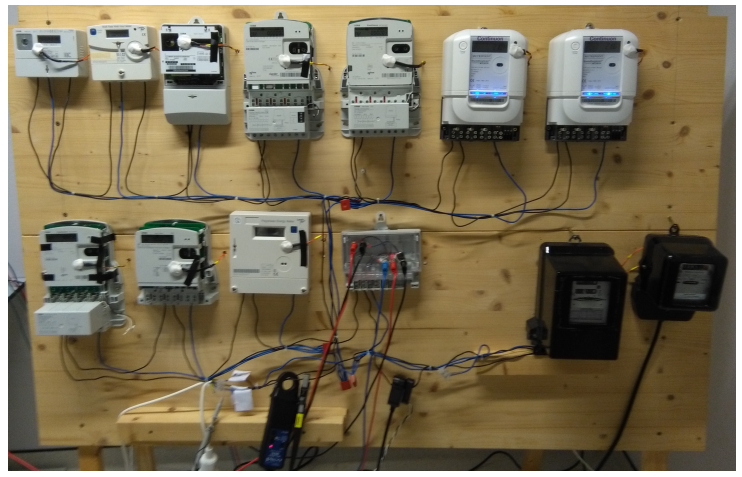

Fig. 8: Ten static meters placed in series.

\section{Measurement Setup}

This section describes the measurement setup that is used for performing the measurements. For the measurements an ideal, non-distorted, single phase sinusoidal voltage is generated using a Pacific Power Smart Source 140-TMX AC power source, which is a 4-quadrant amplifier. A LISN is added to create a stable line impedance, and such that the static meters and load are not disturbed by the interference of a generic and uncontrolled mains supply network. The power generated by the source is connected to a series of ten static energy meters, which can be seen in Fig. 8. The meters that are included in the test setup have different types of current sensors: shunt resistor, current transformer, Hall effect-based current sensor and Rogowski coil. The meters include new and old models and represent the installed base of energy meters in the Netherlands. The energy meter readings of the static meters is corrected with the consumed energy of its following static meters. As load a series of CFL and LED lamps in combination with a power regulator is used. This is the same power regulator as is used in [11], in that paper it is referred to as regulator 1 . These power regulators do not have fixed levels, therefore the power is tuned to the lowest level, for example the signal is dimmed maximum. As the goal of these measurements is to correlate the inclination of the current slope to the static meter deviations, the slope will be changed using a series inductance to the load. The inductance is varied from 0.03 to $1.4 \mathrm{mH}$, which was measured using a Rohde \& Schwarz Hameg HM8118 RLC-bridge at an amplitude of $1 \mathrm{~V}$, frequency of $1 \mathrm{kHz}$, and without bias voltage. Measurements were performed using 7 different inductor values and compared to the situation of no inserted 


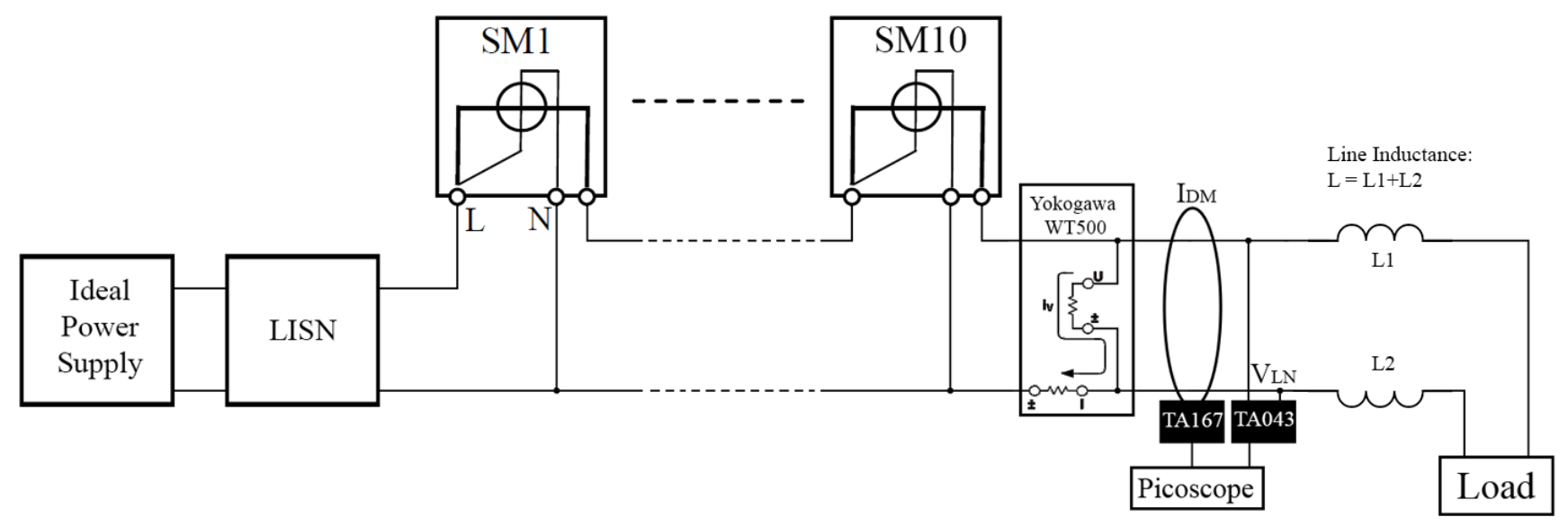

Fig. 9: Schematic overview of the measurement setup.

inductor. The power consumption, as measured by the static meters, is monitored using the method described in [13]. This allows for a faster evaluation of the deviation, as it measures the time-interval between Wh's instead of kWh's to be read manually. A Yokogawa WT500 power analyzer is used as the reference meter for the series of static meters. It has a basic power accuracy of $0.1 \%$ and a sampling rate of $100 \mathrm{kS} / \mathrm{s}$, which should be adequate for reference as we are focused on EMI, and not on metrology. The current and voltage waveform are measured using a Pico Technology TA167 current probe and a Pico Technology TA043 differential voltage probe, which are connected to a Pico Technology Picoscope 4824. Fig. 9 shows a schematic overview of the complete measurement setup.

\section{RESUlts}

This section describes the results of the measurements as described in the previous section. In Fig. 10 the deviations of the static meters with respect to the reference meter are shown, for the different line inductances. The corresponding waveforms generated in these measurements can be seen in Fig. 11. It shows the voltage waveform that is generated using the power supply without a load attached and the current waveforms generated with the different series inductance values. The corresponding characteristics of these waveforms can be seen in Table IV. Without a line inductance, static meter deviations between $-4 \%$ and $+342 \%$ are found. The addition of a line inductance decreases the inclination of the current slope and decreases the static meter deviations. In Fig. 12 the maximum deviations for different static meters are plotted against the current slope. It shows higher deviations if the current slope inclines more.

\section{DISCUSSION}

Several deviations of the static meters are observed during the experiments performed in this paper. In the situation without a line inductance added, static meter deviations between $-4 \%$ and $+342 \%$ are found. In this situation the current slope was $5.6 \mathrm{~A} / \mu \mathrm{s}$. The addition of a line inductance of $0.16 \mathrm{mH}$ resulted in a reduced slope of $0.8 \mathrm{~A} / \mu \mathrm{s}$ and static meter

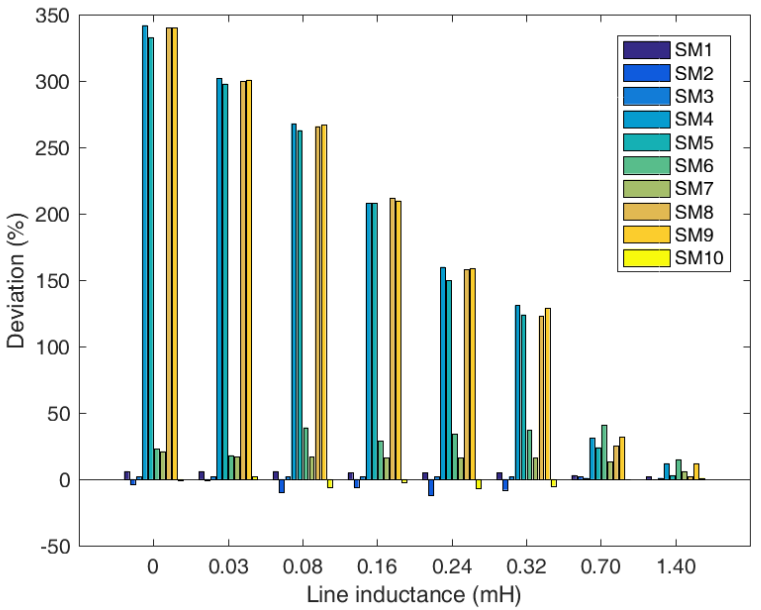

Fig. 10: Deviations [\%] between the static meters (SM) and reference, for different line inductances added.

TABLE IV: Rise time, rising edge and slope of current spikes, for different line inductances (ind.).

\begin{tabular}{|l|r|r|r|}
\hline Line ind. $[\mathrm{mH}]$ & Rise time $[\mu \mathrm{s}]$ & Rising edge $[\mathrm{A}]$ & Slope $[\mathrm{A} / \mu \mathrm{s}]$ \\
\hline 0 & 2.1 & 11.8 & 5.6 \\
\hline 0.03 & 5.6 & 12.0 & 2.1 \\
\hline 0.08 & 10.9 & 12.9 & 1.2 \\
\hline 0.16 & 14.3 & 11.1 & 0.8 \\
\hline 0.24 & 35.9 & 10.2 & 0.3 \\
\hline 0.32 & 43.3 & 9.6 & 0.2 \\
\hline 0.7 & 115.3 & 13.1 & 0.1 \\
\hline 1.4 & 381.8 & 7.0 & 0.02 \\
\hline
\end{tabular}

deviations between $-6 \%$ and $+212 \%$. Reducing the slope even further to $0.02 \mathrm{~A} / \mu \mathrm{s}$, by adding a $1.4 \mathrm{mH}$ line inductance, reduces the meter deviations below $\pm 10 \%$. In Fig. 10 and Table IV also other results with different line impedances are shown, it shows that the deviations and slope decrease for higher inductances. In Fig. 12 it is shown that if the inclination of the current slope is higher, also higher static meter deviations were found. 


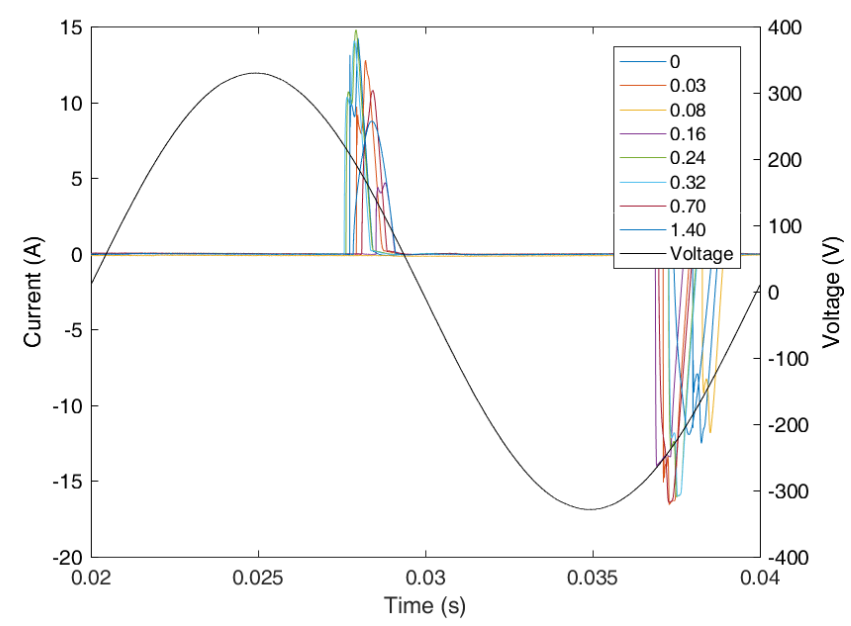

Fig. 11: Current waveforms generated, inserted inductances given in $\mathrm{mH}$, the voltage is the wave generated by the power supply without a load.

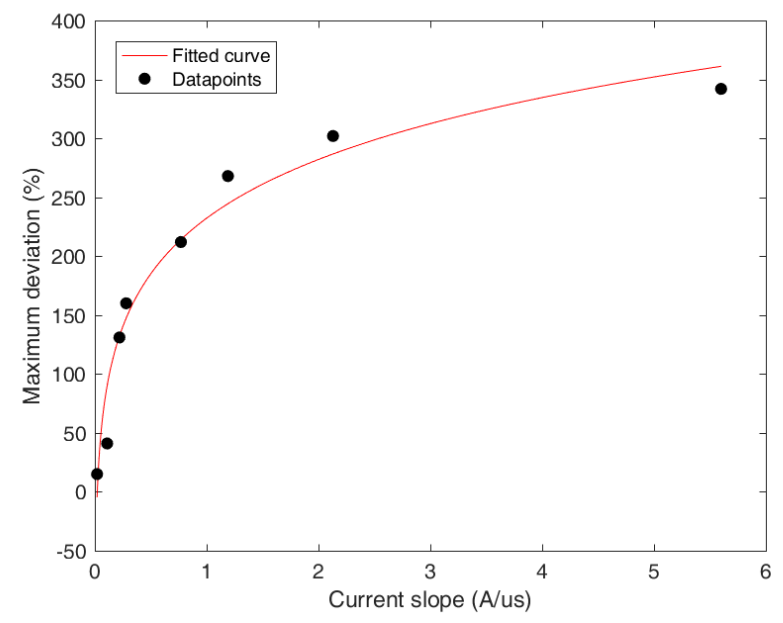

Fig. 12: Correlation between the inclination of the current slope and the maximum static meter deviations.

Next to this observation it was shown, based on previous research, that phase shift and grid impedance has a high effect on the deviations of static meters [10]. A larger phase shift of a current pulse resulted in higher static meter deviations. This can be observed from Fig. 4 and 5, as the maximum deviation at maximum phase shift is $+483 \%$ and almost no deviations are found with the lowest phase shift. Lowering the grid impedance, also increases the static meter deviations. When the grid impedance was changed from the high to low impedance, the maximum deviations increases from $+483 \%$ to $+2114 \%$, Fig. 6 .

\section{CONCLUSION}

In this paper a correlation between the inclination of the current slope and the static meter deviations is shown experimentally. This correlation shows that when the slope is higher, also higher deviations are observed. When a slope of $5.6 \mathrm{~A} / \mu \mathrm{s}$ is generated, deviations between $-4 \%$ and $+342 \%$ are observed. While reducing the slope to $0.02 \mathrm{~A} / \mu \mathrm{s}$, by adding line inductances, deviations below $\pm 10 \%$ have been shown. Measurement points in between these two extremes confirm this correlation even further. Next to this observation static meter deviations can also be caused and even increased by adding phase shift or changing the impedance of the grid, lowering the grid impedance caused higher deviations of static meters.

\section{ACKNOWLEDGMENT}

This project has received funding from the EMPIR programme co-financed by the Participating States and from the European Union's Horizon 2020 research and innovation programme. The results found reflect the author's view only. EURAMET is not responsible for any use that may be made of the information it contains.

\section{REFERENCES}

[1] M. Hossein Pourarab, Morteza; Alishahi, Saeed; Hakkak Sadeghi, “Analysis of harmonic distortion in distribution networks injected by nonlinear loads," 21st International Conference on Electricity Distribution, 2011.

[2] F. Leferink, "Conducted interference, challenges and interference cases," IEEE Electromagnetic Compatibility Magazine, vol. 4, no. 1, pp. 78-85, 2015.

[3] R. Quijano Cetina, A. J. Roscoe, and P. S. Wright, "Challenges for Smart Electricity Meters due to Dynamic Power Quality Conditions of the Grid: A Review," AMPS 2017 - IEEE International Workshop on Applied Measurements for Power Systems, Proceedings, 2017.

[4] R. Masnicki, "Some Remarks on the Accuracy of Energy Meters," 2018 IEEE International Conference on Environment and Electrical Engineering and 2018 IEEE Industrial and Commercial Power Systems Europe (EEEIC / I\&CPS Europe), 2018.

[5] J. Kirchhof and G. Klein, "EMV - Grenzwertlücke Wechselrichter stört Zähler," 24. Symposium Photovoltaische Solarenergy, Bad Staffelstein, 2009.

[6] J. Kirchhof, “Grenzwertlücke - Wechselrichter stört Elektrizitätszähler," EMV, Düsseldorf, 2010.

[7] F. Leferink, C. Keyer, and A. Melentjev, "Runaway energy meters due to conducted electromagnetic interference," IEEE International Symposium on Electromagnetic Compatibility, pp. 172-175, 2016.

[8] F. Leferink, C. Keyer, and A. Melentjev, "Static energy meter errors caused by conducted electromagnetic interference," IEEE Electromagnetic Compatibility Magazine, vol. 5, no. 4, pp. 49-55, 2016.

[9] C. Keyer and F. Leferink, "Conducted interference on smart meters," IEEE International Symposium on Electromagnetic Compatibility, pp. 608-611, 2017.

[10] B. Have, T. Hartman, N. Moonen, C. Keyer, and F. Leferink, "Faulty Readings of Static Energy Meters Caused by Conducted Electromagnetic Interference from a Water Pump,' Submitted to Renewable Energy and Power Quality Journal (RE\&PQJ), 2019.

[11] B. Have, T. Hartman, N. Moonen, and F. Leferink, "Misreadings of Static Energy Meters due to Conducted EMI caused by Fast Changing Current," Submitted to 2019 Joint International Symposium on Electromagnetic Compatibility and Asia-Pacific International Symposium on Electromagnetic Compatibility, 2019.

[12] E. M. Chery and D. E. Hooper, "Amplifying Devices and Low-Pass Amplifier Design,” IEEE Spectrum, vol. 6, no. 3, pp. 134-143, 1969.

[13] B. Have, C. Keyer, and F. Leferink, "Monitoring of Power Measured by Static Energy Meters for Observing EMI Issues," 2018 International Symposium on Electromagnetic Compatibility (EMC Europe), pp. 903 907, 2018. 